\title{
Beliefs about Causes of and Risk Factors for Mental Disorders: A Comparison of Japanese and American College Students
}

\author{
Niwako Yamawaki ${ }^{1}$, Christina Riley ${ }^{1}$, Takeshi Sato $^{2} \&$ Mika Omori $^{3}$ \\ ${ }^{1}$ Brigham Young University, Department of Psychology, Utah, U.S.A. \\ ${ }^{2}$ Saga University, Health Care Center, Japan \\ ${ }^{3}$ Ochanomizu University, Department of Psychology, Tokyo, Japan \\ Correspondence: Niwako Yamawaki, Department of Psychology, Brigham Young University, 1094 SWKT \\ Provo Utah, 84602, United States. Tel: 801-422-8053. E-mail: Niwako_yamawaki@byu.edu
}

Received: January 16, 2015 Accepted: February 5, 2015 Online Published: May 15, 2015

doi:10.5539/ass.v11n15p197 URL: http://dx.doi.org/10.5539/ass.v11n15p197

\begin{abstract}
Objective: Patterns of mental health literacy in depression between college students in the United States and Japan were examined. Participants: 289 American students and 298 Japanese students were recruited. Methods: Students read a scenario in which a man presents the symptoms of major depression and completed surveys that measured the ability to identify mental illness, beliefs in helpfulness of interventions, and described previous experience with depression. Results: National and gender differences were found in the ability to correctly recognize depression, beliefs in the cause of the depression, and recommended intervention. An interaction effect of country and gender was found for recommended intervention. Path-analytic mediation analyses showed that the national differences in recognition for depression were mediated by the national differences in experience with depression. Conclusions: Implications of the national and gender differences in MHL on the utilization of mental health services are discussed.
\end{abstract}

Keywords: mental health literacy, cross-cultural, knowledge of depression

\section{Background}

Depression is becoming increasingly prevalent among college students. One nationwide survey found that nearly 30 percent of college students reported severe depression within the past year, and that number is only getting larger (National Institutes of Mental Health [NIMH], 2011). Other studies have found that up to 53\% of college students report experiencing depression at some point during their college years (Lindsey, Fabiano, \& Stark, 2009). College students have a high rate of early-onset mood disorders, which have a more malignant course and greater comorbidity than do their late-onset counterparts (Klein et al., 1999). Due to the adverse side effects of depression, including impaired academic and social functioning, increased drug use, and increased risk of suicide (Lindsey et al., 2009), early detection and treatment of depression is critical.

College students are particularly susceptible to depression (Lindsey et al., 2009; National Institute of Mental Health, 2011); thus, it is important that they are knowledgeable about mental disorders so that they can better recognize the symptoms and seek proper treatment. This type of knowledge concerning mental disorders is known as Mental Health Literacy (MHL). MHL. Jorm et al. (1982) defined MHL as the "knowledge and beliefs about mental disorders which aid their recognition, management, or prevention" (p. 182). Some researchers concluded that college students are particularly vulnerable to mental disorders, specifically depression (e.g., Lindsey et al., 2009). However, depression is often undiagnosed, and therefore goes untreated (Coles, Coleman, \& Heimberg, 2008). One explanation for this lack of attention is that the symptoms can be mislabeled as a normal part of this transition period. Accurate MHL of depression during the early college years can keep students from becoming overwhelmed, promote destigmatization of the disorder (Canadian Alliance on Mental Illness and Mental Health, 2007), and encourage students to seek professional help (Coles et al., 2008).

The cultural effects on MHL should also be taken into account since research shows a strong cultural impact on the classification and treatment of mental disorders (Carpenter-Song et al., 2010; Courtis, Lauber, Trujillo Costa, \& Cattapan-Ludewig, 2008; Good, James, Good, \& Becker, 2003). The cultural differences in MHL can have negative impacts, including an individual with a mental disorder not being identified as needing mental health 
care, or as an individual not receiving needful help from a trained mental health care professional (Sartorius, 2007). There are many cultural factors that affect one's capacity for MHL. Industrialization (Levine, 2005), cultural beliefs on sources of mental illness (Arkoff, Thver, \& Elkind, 1966), and expected coping strategies of mental and emotional struggles have all been found to differ by culture (Inman \& Yeh, 2007). For instance, Eastern cultures, more than Western cultures, are much more likely to describe the source of a mental illness as the result of organic factors, a lack of individual will power, and morbid thinking (Sue, Wagner, Ja, Margullis, \& Lew, 1976). Placing emphasis on such factors as these creates a cultural expectation on members of Eastern cultures to suppress and accepts ones' problems instead of facing them and to preserve personal and public identity by not revealing problems to others (Morling, Kitayama, \& Miyamoto, 2002; Tweed \& Conway, 2006; Yeh, Ahora, \& Wu, 2006).

Cultural expectations towards mental health in Eastern societies have been shown to suppress peoples' desire to seek mental health services (Yeh, Inman, Kim, \& Okubo, 2006). Not only do individuals try to avoid seeking help for mental illness, but the capacity of accurate MHL by properly identifying an individual's need of mental health care may be compromised in Eastern societies as well (Rong et al., 2009). Rong et al. (2009) found that medical students in Western and Eastern societies differ in both their correct identification of mental disorders and their attitudes about the severity of the impact of these mental disorders on both the patient and on society in general. Medical students in a Western society were better able to identify persons in need of mental health care compared to those from an Eastern society. Given the important cultural differences in MHL between Western and Eastern cultures, the majority of past studies were conducted among adult populations.

For these reasons, the purpose of this study was to compare the patterns of MHL between the United States and Japan. This study differs from past research (e.g., Nakane et al, 2005), which examined cross-national differences in MHL between Australians and Japanese. The present study investigated MHL differences between Japanese and American using college students rather than general populations. The present investigation is worthy of study for a couple of reasons. First, the identification of early-onset depression is particularly crucial. Klein et al. (1999) found that early-onset chronic major depression (prior to age 21) has a more malignant course and is associated with greater comorbidity than late-onset (after age 35) chronic major depression. Second, college students have been identified as one of the most vulnerable populations for the early onset of mood disorders, and, third, the prevalence of mental illness in college populations has been steadily increasing (Klein et al., 1999). This is significant in that it can direct the ways in which proper education and resources are provided for college students to help prevent their risk of depression. Therefore, the purpose of this study was to investigate the differing patterns in knowledge and beliefs in depression between Japanese and American college students.

\section{Method}

\subsection{Participants}

A total of 289 American students (102 men and 187 women) and 298 Japanese students (143 men and 155 women) participated in this study. American participants were recruited from an undergraduate student research pool in the History Department at a public university in Texas and from undergraduate students taking introductory political science classes at a private university in Utah. Japanese participants were recruited from among students who were enrolled in the College of Agriculture at a public university in the Saga prefecture and students who were taking Accounting class at private university in the Kochi prefecture. For Japanese participants, the average age was 18.8 years, ranging from 18 to 60 and $96 \%$ of them were single. For American participants, the average age was 19.4 years, ranging from 18 to 53 years and $90 \%$ of them were single. Among American participants, $92 \%$ were identified themselves as Caucasian, $5 \%$ Hispanics, and 1\% Blacks, and 2\% others. International students were excluded from both Japanese and American data.

\subsection{Materials}

Scenario. A very brief scenario was modified from Jorm's study and used for the present study to make appropriate for college participants. It describes an individual who displays symptoms that satisfies the diagnostic criteria for major depression according to the Diagnostic and Statistical Manual-IV (DSM-IV), which is used by both American and Japanese healthcare professionals. The scenario describes as follows.

Jon (Kenji) is a 20 years old college student. He has been feeling unusually sad and blue for the last two weeks. $\mathrm{He}$ is always tired, but he wakes up during the night and has difficulty retuning to sleep. Jon (Kenji) has been eating a lot recently and has gained a significant amount of weight. He can't keep his mind on his class work and puts off making decisions. Even day-to-day tasks seem too much for him. This has come to the attention of his roommates, who are concerned about Jon's lowered school performance. 
Mental Health Literacy Scale: Identification Subscale. This subscale was developed by Jorm et al. (2000) and modified to fit the college population in the present study. It purports to measure one's ability to identify mental illness. The items ask whether Jon (Kenji) is suffering from (a) physical illness; (b) mental illness; and (c) stress. Participants rated all items on scales that range from 1 (strongly disagree) to 5 (strongly agree). Then participants chose only one item from lists of mental illnesses that Jon (Kenji) is experiencing. They are (a) mental retardation, (b) depression, (c) schizophrenia, (d) ADHD, (e) learning disorder, (f) eating disorder, (g) others, and (h) don't know. For the purpose of this study, the data was coded 1 for correct identification when participants selected depression and coded 2 for incorrect recognition when they selected other mental illness. Because some studies reported that previous experience with mental illness was associated with positive attitudes toward mental illness, participants were asked whether or not they have met someone close to them who experience depressive symptoms like Jon (Kenji) or whether they themselves have experienced the same problem. They were asked to answer "yes $=1$ "or "no $=2$." In addition, participants were also requested to estimate the prevalence of depression by responding to the question, "What percentage of students in your university do you estimate have these kinds of problems?"

Mental Health Literacy Scale: Best Method of Help Subscale. This subscale is designed to measure the respondents' beliefs in the likely helpfulness of interventions. Although Jorm et al. (2000) used open ended questions about how the person in the vignette could best be helped, this studyprovide a list of methods of help based on their findings and that were appropriate for college age students. The participants were asked to rate their agreement using a Likert-type scale, ranging from 1 (strongly disagree) to 5 (strongly agree) for the following items: (a) take medication, (b) see a counselor or receive counseling, (c) talk with friends/family, and (d) see non-professionals, such as fortune teller, religious leaders, etc.

\section{Results}

Recognition of depression. Chi-square analyses were performed to investigate the national and gender differences in correctly recognizing depression from the symptoms described in the scenario. The results showed that American or female college students were more likely than Japanese or male college students to correctly recognize depression $\left[X^{2}(1, N=587)=167.09, p<.001\right.$; and $X^{2}(1, N=587)=14.22, p<.0001$, respectively $]$. Table 1 represents the percentage of participants who answered given diagnoses.

Table 1. Percentage of participants who answered given diagnoses

\begin{tabular}{ccccc}
\hline & \multicolumn{2}{c}{ America } & \multicolumn{2}{c}{ Japan } \\
\cline { 2 - 5 } & Male & Female & Male & Female \\
\hline Mental retardation & $1(1 \%)$ & 0 & $19(13.3 \%)$ & $15(9.7 \%)$ \\
Depression & $89(87.3 \%)$ & $170(90.9 \%)$ & $45(31.5 \%)$ & $69(44.5 \%)$ \\
Schizophrenia & 0 & 0 & $8(5.6 \%)$ & $3(1.9 \%)$ \\
ADHD & 0 & $2(1.1 \%)$ & $4(2.8 \%)$ & $3(1.9 \%)$ \\
LD & 0 & $1(.5 \%)$ & 0 & $1(.6 \%)$ \\
Eating disorder & 0 & $1(.5 \%)$ & $10(7 \%)$ & $11(7.1 \%)$ \\
Others & $1(1 \%)$ & $1(.5 \%)$ & $8(5.6 \%)$ & $4(2.6 \%)$ \\
Don't know & $11(10.8 \%)$ & $12(.5 \%)$ & $49(34.2 \%)$ & $49(31.7 \%)$ \\
\hline
\end{tabular}

In order to test respondents' beliefs of what Jon's (Kenji's) problem is, a 2 (country) x 2 (participant's gender) MANOVA on physical illness, mental illness, and stress was performed. The results showed that there was a main effect of country $[F(3,580)=129.63, p<.001]$. A follow up ANOVA showed that American participants, more than Japanese participants, tended to believe that Jon's (Kenji's) problem was mental illness $[F(1,582)=$ $564.55, p<.001]$. On the contrary, Japanese respondents tended to believe that Jon's (Kenji's) problem was the result of physical illness or stress more than did American respondents $[F(1,582)=129.63, p<.001$, and $F(1$, $582)=92.47, p<.001$, respectively]. There was no main effect of participants' gender or interaction effect.

Estimation of the prevalence of depression. Participants were asked to estimate the percent of college students who are suffering from Jon's (Kenji's) symptoms. To investigate the difference in this estimation, a two-way (country and participant's gender) ANOVA was performed. The results showed that there were main effects of country and participant's gender $[F(1,565)=349.09, p<.001$ and $F(1,565)=25.76, p<.001$, respectively $]$. That is, Japanese $(\mathrm{M}=11.61, \mathrm{SD}=9.99)$ or male participants $(\mathrm{M}=20.52, \mathrm{SD}=14.06)$ were more likely to underestimate the occurrence of depression more than American $(\mathrm{M}=35.12, \mathrm{SD}=18.65)$ or female participants $(\mathrm{M}=27.21, \mathrm{SD}=14.69)$. 
Recommended Best Method of Help. To examine the national and gender differences on the recommended method of help, a 2 (country) x 2 (participant's gender) MANOVA was performed on the medication, counseling, consultation with family, and consultation with non-professionals. The results showed that there were main effects of country, gender, and an interaction effect of country x gender $[F(4,580)=66.04, p<.001 ; F(4,580)$ $=11.59, \mathrm{p}<.001$; and $\mathrm{F}(4,580)=5.21, \mathrm{p}<.001$, respectively]. A follow-up ANOVA showed that American participants tended to recommend drug treatment and counseling more than did Japanese participants $[\mathrm{F}(1,583)$ $=86.83, \mathrm{p}<.001 ; \mathrm{F}(1,583)=77.83, \mathrm{p}<.001$, respectively], while Japanese participants recommended to consult with non-professionals more than did American participants $[\mathrm{F}(1,583)=99.95, \mathrm{p}<.001]$. Furthermore, female participants were more likely to recommend counseling and consulting with family than were male participants $[\mathrm{F}(1,583)=32.45, \mathrm{p}<.001 ; \mathrm{F}(1,583)=20.39, \mathrm{p}<.001$, respectively $]$.

Prevalence of and Experience with Depression. To investigate participants' estimate for prevalence of depression, a two-way ANOVA (country and gender of participants) was conducted. The results indicated that Japanese and male students were inclined to estimate the prevalence of depression significantly less than American and female students did $[\mathrm{F}(1,565)=349.09, \mathrm{p}<.001 ; \mathrm{F}(1,565)=25.77, \mathrm{p}<.001$, respectively]. Furthermore, chi-square tests were performed on whether respondents have known someone like Jon (Kenji) or whether they themselves have experienced Jon's (Kenji's) problem. The result indicated that American or female participants tended to have experienced themselves or to know someone who has experienced symptoms of depression more than did Japanese or male participants $\left[\mathrm{X}^{2}(1, \mathrm{~N}=564)=89.56, \mathrm{p}<.001 . \mathrm{X}^{2}(1, \mathrm{~N}=557)=8.76, \mathrm{p}<.05\right.$, respectively]. Another chi-square test was also conducted to examine the relationship between correct recognition for depression and experience with depression. The results showed that people who know someone with depression or who have experienced depression were inclined to correctly recognize that Jon (Kenji's) problem was indeed depression $\left[\mathrm{X}^{2}(1, \mathrm{~N}=576)=37.31, p<.001\right]$.

In addition, path-analytic mediation analyses were performed using the guidelines developed by Baron and Kenny (1986). To achieve a mediating effect, three requirements must be met. First, the predictor variable (nation) must show a significant relationship with both the mediator (experience in depression) and the criterion variable (recognizing depression). Second, the mediator must show a significant relationship with the criterion variable. Finally, when the relationship between the mediator and the criterion is held constant, the previously significant relationship between the predictor variable and the criterion variable should be significantly reduced. Sobel's significance test was used to analyze the indirect effect of the predictor variable on the criterion via the mediator. The results demonstrated that all three relationships - (a) country and experience with depressive symptoms, (b) experience with depression and recognition, and (c) country and recognition, were significant (b $=.36 ;-.68$; and $-.182, \mathrm{p}<.001$, respectively). Sobel's test for indirect effect was also performed, and the results showed that the national differences in recognition for depression was mediated by the national difference in experience with depression $(\mathrm{p}<.001)$.

\section{Discussion}

The purpose of this cross-cultural study was to examine the differing patterns in college students' ability to recognize the symptoms of depression, beliefs in treatment modality, and how their experience with depression influence their ability to correctly recognize and identify depression. One of the notable findings from this study was the effects of country and participants' gender on MHL. That is, in comparison of Japan and the United States and male and female participants, American or female participants were more likely than were Japanese or male participants to recognize depression from the scenario, had more experience with depression or know someone who has had depression, estimated greater prevalence of depression, and chose counseling for dealing with depression. Furthermore, American students were significantly more likely than were Japanese students to deal with depression by talking to non-professionals, such as fortunetellers.

Recognizing mental illness is the first step to seeking help from mental health professionals and receive social support. However, the results of this study indicated that Japanese or male participants tended not to recognize the symptoms of depression. Such failure of recognition may be related to the current problems of underutilization of mental health services by Asians, including Japanese, and males in general in comparison to Americans or females. As for the national difference in recognition, for instance, Naganuma et al. (2006) found that the majority of Japanese participants with psychiatric disorders did not access mental health care or other support systems, despite the fact that the mental health care system in Japan has improved over the past decade. Tomoda et al. (2000) reported high prevalence of depression among Japanese college students and suggested that they may be vulnerable to depression due to adjustment to a new college environment. Given that the high prevalence of depression and experience of a depressive episode early in life without getting treatment greatly increases the risk of further depressive episodes and co-occurring psychiatric disorders (Harrington, Fudge, 
Rutter, Pickles, \& Hill, 1990; Kashani, Carlson, Beck, et al., 1987; Kovacs, Feinber, Crouse-Novak, Paulauskas, \& Finkelstein, 1984), it is crucial to educate students about the symptoms of depression. College students, in general, are likely to experience the stress and anxiety of attending college, leaving home, developing new relationships and the pressure to succeed. Furthermore, Arehart-Treichel (2002) warned that the peak age of onset of depression is college-age and Fisher (2004) concluded that mood disorders that occur in college might be life-threatening. Therefore, early intervention should be seriously considered for young adults, especially when they enter college.

The results of this study also indicate that experiencing depression or knowing someone with depression was a crucial factor students in their ability to recognize the symptoms of depression as mental illness and identify the symptoms as depression. That is, the national difference in recognizing depression was mediated by the national difference in such experience. This finding may be explained by the fact that it is still discouraged for Japanese individuals to discuss mental illness in public. In particular, talking about their own experiences of depression and its symptoms, in public, is extremely difficult for them because of the fear of negative evaluation by others. For instance, Maekawa and Kanai (2015) found that one unique psychological factor, called sekentei, was a significant barrier to seeking professional help. Sekentei, or "appearance in the eyes of others" (Maruyama et al., 2011), is a key aspect of Japanese culture and one of the main features of Japanese behavioral principles. Therefore, Japanese college students, in comparison to American students, may be less likely to talk about the symptoms and experiences of their own depression, in public, in order to avoid shame, to keep up "sekentei." Furthermore, American college students reported knowing someone with depression or experiencing depression themselves more than Japanese students did, despite the similar prevalence of depression in both countries. This tendency may also be associated with the stigma Japanese individuals face when discussing mental illness. Indeed, Nakane et al. (2005) concluded that Japanese individuals tended to believe that depression is caused by weakness of character. Furthermore, very few Japanese believe that people can recover from mental disorders, such as depression (Ando, Yamaguchi, Aoki, \& Thomickroft, 2013). Therefore, it may be significantly more difficult for Japanese college students to disclose that they are experiencing a mental disorder than it is for American college students to do so. Furthermore, the poor MHL of males may be associated with cultural norms of traditional masculine stereotype; that is, men are expected to be self-reliant, stoic, and emotionally in control (Yamawaki, 2010). Such roles may lead males to be reluctant to disclose that they are experiencing depression, which results in poor MHL.

Similar to findings of previous studies, Japanese individuals were less likely to utilize counseling or medications in comparison to American participants (Nakene et al., 2005). An examination of systematic reviews and meta-analyses of research comparing the effectiveness of antidepressants and counseling for individuals with depression has shown that counseling is as effective as antidepressants for mild to moderate depression and may be equally effective for chronic or severe depression as well (Hagen, Wong-Wylie, \& Pijl-Zieber, 2010). Thus, it is imperative for Japanese mental health professionals to educate college students about the effectiveness of such treatment modalities. Further, future research studies are necessary to investigate the barriers to receiving counseling and medication treatment. Interestingly, Japanese college students were inclined to talk to non-professionals for depressive symptoms. This may be due to the fact that Japanese students often resist seeking help because of the potential negative stigma of counseling and medication treatment (Yoo, 2002). Thus, rather than seeking professional help, they may tend to talk to non-professionals. In addition, Jorm et al. (2005) found a national difference for talking to friends/family between Japanese and Australian participants. Japanese participants tended to talk to their friends and families more than Australian participants. However, the present study could not replicate such a finding. This may be associated with the study by Jorm et al. (2005) comparing Japanese and Australian participants, while the present study compared American and Japanese individuals. However, we believe that this inconsistency is more likely associated with a developmental issue. For instance, developmentally, in comparison to the general public, college students are still well-connected with their families and friends. Such a connection may influence the similar patterns in seeking help.

We have to note that this study is not free from limitations. For instance, it is imperative to collect representative data from college students in varied regions from both countries. Furthermore, more comprehensive questions, such as investigating cultural stigma relating to mental illness and help-seeking or a greater number of methods of help, should be included to support our claim.

\section{References}

Ando, S., Yamaguchi, S., Aoki, Y., \& Thomicroft, G. (2013). Review of mental health related stigma in Japan. Psychiatry and Clinical Neuroscience, 67, 471-482. http://dx.doi.org/10.1111/pcn.12086 
Arehart-Treichel, J. (2002, March 15). Mental Illness on rise on college campuses. Psychiatric News, 37(6). http://dx.doi.org/10.1176/pn.37.6.0006

Arkoff, A., Thaver, F., \& Elkind, L. (1966). Mental health and counseling ideas of Asian and American students. Journal of Counseling Psychology, 13, 219-223. http://dx.doi.org/10.1037/h0023396

Baron, R. M., \& Kenny, D. A. (1986). The moderator-mediator variable distinction in social psychological research: Conceptual, strategic, and statistical considerations. Journal of Personality and Social Psychology, 51, 1173-1182. http://dx.doi.org/10.1037/0022-3514.51.6.1173

Carpenter-Song, E., Chu, E., Drake, R. E., Ritsema, M., Smith, B., \& Alverson, H. (2010). Ethno-cultural variations in the experience and meaning of mental illness and treatment: Implications for access and utilization. Transcultural Psychiatry, 47(2), 224-251. http://dx.doi.org/10.1177/1363461510368906

Coles, M., Coleman, S., \& Heimberg, R. (2008). Addressing patient needs: The role of mental health literacy. American Journal of Psychiatry, 165(3), 399. http://dx.doi.org/10.1176/appi.ajp.2007.07101648

Courtis, D. N., Lauber, L., Trujillo Costa, C., \& Cattapan-Ludewig, K. (2008). Beliefs about the mentally ill: A comparative study between healthcare professionals in Brazil and Switzerland. International Review of Psychiatry, 20(6), 503-509. http://dx.doi.org/10.1080/09540260802565125

Fisher, S. (2004). American college health association survey shows increase of depression among college students over four year period. American College Health Association. Retrieved from http://www.acha.org/ newsroom/pr ncha_11_17_04.cfm

Furnham, A., \& Murao, M. (1999). A cross-cultural comparison of British and Japanese lay theories of Schizophrenia. International Journal of Social Psychiatry, 46, 4-20. http://dx.doi.org/10.1177/002076 400004600103

Good, M., James, C., Good, B., \& Becker, A. (2003). The culture of medicine and racial, ethnic, and class disparities in healthcare. In B. D. Smedley, A. Y. Stith, \& A. R. Nelson (Eds.), Unequal treatment: Confronting racial and ethnic disparities in health care. Washington, DC: National Academies Press.

Hagen, B., Wong-Wylie, G., \& Wong-Wylie, E. (2010). Tablets or talk? A critical review of the literature comparing antidepressants and counseling for treatment of depression. Journal of Mental Health counseling, 32, 102-124.

Harrington, R., Fudge, H., Rutter, M., Pickles, A., \& Hill, J. (1990). Adult outcomes of childhood adolescent depression. Archive of General Psychiatry, 47, 465-473. http://dx.doi.org/10.1001/archpsyc.1990.01810 170065010

Inman, A. G., \& Yeh, C. (2007). Stress and coping. In F. T. L. Leong, A. G. Inman, A. Ebreo, L. Lang, L. Kinoshita, \& M. Fu (Eds.), Handbook of Asian American psychology (2nd ed., pp. 323-340). Sage.

Jorm, A. F. (2000). Mental health literacy: Public knowledge and beliefs about mental disorders. British Journal of Psychiatry, 34, 396-401. http://dx.doi.org/10.1192/bjp.177.5.396

Jorm, A. F., Korten, A. E., Jacomb, P. A., Christensen, H., Rodgers, B., \& Pollitt, P. (1997). "Mental health literacy": A survey of the public's ability to recognize mental disorders and their beliefs about the effectiveness of treatment. Medical Journal of Australia, 166.

Jorm, A. F., Nakane, Y., Christensen, H., Yoshioka, K., Griffiths, K. M., \& Wata, Y. (2005). Public beliefs about treatment and outcome of mental disorders: a comparison of Australia and Japan. Bio Medical Central Medicine, 3, 1-14.

Kashani, J. H., Carlson, G. A., \& Beck, N. C. (1987). Depression, depressive symptoms, and depressed mood among a community sample of adolescents. American Journal of Psychiatry, 144, 931-934. http://dx.doi.org/10.1176/ajp.144.7.931

Klein, D. N., Schatzberg, A. F., McCullough, J. P., Dowling, F., Goodman, D., \& Howland, R. H. (1999). Age of onset in chronic major depression: Relation to demographic and clinical variables, family history, and treatment response. Journal of Affective Disorders, 55, 149-157. http://dx.doi.org/10.1016/S01650327(99)00020-8

Kovacs, M., Feinber, T. L., Crouse-Novak, M. A., Paulauskas, Sl. L., \& Finkelstein, R. (1984). Depressive disorders in childhood: A longtitudinal prospective study of characteristics and recovery. Archive of General Psychiatry, 41, 229-237. http://dx.doi.org/10.1001/archpsyc.1984.01790140019002 
Levine, B. E. (2005). Mental illness or rebellion? Ethical human psychology and psychiatry. An International Journal of Critical Inquiry, 7(2), 125-129.

Lindsey, B. J., Fabiano, P., \& Stark, C. (2009). The prevalence and correlates of depression among college students. College Student Journal, 43, 999-1014.

Maekawa, Y., \& Kanai, A. (2015). Effects of Sekentei on seeking psychological help in Japan: The interaction effects of moderating factors based on the theory of reasoned action. Online Journal of Japanese Clinical Psychology, 1, 1-12

Maruyama, H., Taguchi, A., Ryu, S., Nagata, S., \& Murashima, S. (2011). Is sekentei associated with attitudes toward use of care services? Multilevel analysis in Japan. Geriatrics \& Gerontology International, 11, 166-173. http://dx.doi.org/10.1111/j.1447-0594.2010.00658.x

Morling, B., Kitayama, S., \& Miyamoto, Y. (2002). Cultural practices emphasize influence in the U.S. and adjustment in Japan. Personality and Social Psychology Bulletin, 28, 311-323.

Naganuma, Y., Tachimori, H., Kawakami, N., Takeshima, T., Ono, Y., \& Uda, H. (2006). Twelve-month use of mental health services in four areas in Japan: Findings from the world mental health Japan survey. Psychiatry and Clinical Neuroscience, 60, 240-248. http://dx.doi.org/10.1111/j.1440-1819.2006.01492.x

Nakane, Y., Jorm, A. F., Yoshioka, K., Christensen, H., Nakane, H., \& Griffiths, K. (2005). Public beliefs about causes and risk factors for mental disorders: A comparison of Japan and Australia. Bio Medical Central Medicine, 5, 1-9. http://dx.doi.org/10.1186/1471-2288-5-1

National Institutes of Mental Health. (2011). Depression and college students: How does depression affect college students? Retrieved November 5, 2011, from http://www.nimh.nih.gov/health/publications/ depression-and-college-students/q-how-does-depression-affect-college-students.shtml

Rong, Y., Luscombe, G. M., Davenport, T. A., Huang, Y., Glozier, N., \& Hickie, I. B. (2009). Recognition and treatment of depression: A comparison of Australian and Chinese medical students. Social Psychiatry and Psychiatric Epidemiology, 44(8), 636-642. http://dx.doi.org/10.1007/s00127-008-0471-5

Sartorius, N. (2007). Stigma and mental health. Lancet, 370(9590), 810.

Sue, S., Wagner, N., Ja, D., Margullis, C., \& Lew, L. (1976). Conceptions of mental illness among Asian and Caucasian-American students. Psychological Reports, 38, 703. http://dx.doi.org/10.2466/pr0.1976.38.3.703

The Canadian Mental Health Alliance on Mental Illness and Mental Health. (2007). Mental health literacy: A review of the literature. Ottawa, ON: CAMIMH. (Original work published 2004).

Tomoda, A., Mori, K., Kimura, M., Takahashi, T., \& Kitamura, T. (2000). One-year prevalence and incidence of depression among first-year university students in Japan: A preliminary study. Psychiatry and Clinical Neuroscience, 54, 583-588. http://dx.doi.org/10.1046/j.1440-1819.2000.00757.x

Tweed, R. G., \& Conway, L. G., III. (2006). Coping strategies and culturally influenced beliefs about the world. In P. T. P. Wong, \& L. C. J. Wong (Eds.), Handbook of multicultural perspectives on stress and coping: International and cultural psychology series (pp. 133-153). Dallas: Spring.

Yamawaki, N. (2010). The effects of self-construals and masculinity vs. femininity: A comparison of American and Japanese attitudes toward mental health services. Journal of Mental Health Counseling, 32, 154-167.

Yeh, C. J., Arora, A. K., \& Wu, K. A. (2006). A new theoretical model of collectivistic coping. In P. T. P. Wong, \& L. C. J. Wong (Eds.), Handbook of multicultural perspectives on stress and coping: International and cultural psychology series (pp. 55-72). Dallas: Spring.

Yeh, C. J., Inman, A. C., Kim, A. B., \& Okubo, Y. (2006). Asian American families' collectivistic coping strategies in response to 9/11. Cultural Diversity and Ethnic Minority Psychology, 12(1), 134-148. http://dx.doi.org/10.1037/1099-9809.12.1.134

Yoo, S. K. (2002, October). Psychological and cultural antecedents to attitudes toward seekingprofessional psychological help: Implications for counseling teacher education. Paper presented at Third International Conference on Education Research, Seoul National University, Seoul, Korea.

\section{Copyrights}

Copyright for this article is retained by the author(s), with first publication rights granted to the journal.

This is an open-access article distributed under the terms and conditions of the Creative Commons Attribution license (http://creativecommons.org/licenses/by/3.0/). 